\title{
CHANGE DETECTION IN REMOTE SENSING IMAGES USING CONDITIONAL ADVERSARIAL NETWORKS
}

\author{
M. A. Lebedev ${ }^{1, *}$, Yu. V. Vizilter ${ }^{1}$, O. V. Vygolov ${ }^{1}$, V. A. Knyaz ${ }^{1}$, A. Yu. Rubis ${ }^{1}$ \\ ${ }^{1}$ State Res. Institute of Aviation Systems (GosNIIAS) - (MLebedev, viz, o.vygolov, knyaz)@ gosniias.ru, arcelt@mail.ru
}

\author{
Commission II, ICWG II/III
}

KEY WORDS: Change Detection, Database, Deep Convolutional Neural Networks, Generative Adversarial Networks

\begin{abstract}
:
We present a method for change detection in images using Conditional Adversarial Network approach. The original network architecture based on pix2pix is proposed and evaluated for difference map creation. The paper address three types of experiments: change detection in synthetic images without objects relative shift, change detection in synthetic images with small relative shift of objects, and change detection in real season-varying remote sensing images.
\end{abstract}

\section{INTRODUCTION}

Change detection in the time-varying sequences of remote sensing images acquired on the same geographical area is an important part of many practical applications, e.g. urban development analysis, environmental inspection, agricultural monitoring. In most cases, solving the change detection task in manual mode is a highly time-consuming operation, which makes an automation of this process an important and practically demanded filed of research.

At present, the best results in the overwhelming majority of image analysis and processing tasks are delivered by methods based on deep convolutional neural networks $(\mathrm{CNN})$. In this paper, we propose a new method for automatic change detection in season-varying remote sensing images, which employs such a modern type of CNN as Conditional Adversarial Networks.

\section{RELATED WORKS}

A lot of change detection techniques are developed for remote sensing applications (Singh et al., 1989; Lu et al. 2004; Chen et al. 2013; Hussain et al. 2013). In (Hussain et al. 2013) two main categories of methods are pointed: pixel-based change detection (PBCD) and object-based change detection (OBCD). The PBCD category contains the direct, transform-based, classificationbased and learning-based comparison of images at the pixel level (Wiemker, 1997; Bruzzone and Fernandez-Prieto, 2002; Ghosh et al., 2007; Benedek and Szir'anyi, 2009; Singh et al., 2014; Rubis et. al., 2016). The OBCD category contains direct, classified and composite change detection at the object level (Liu and Prinet, 2006; Castellana et al., 2007; Zhong and Wang, 2007; Szir'anyi and Shadaydeh, 2014). We start our brief overview from $\mathrm{PBCD}$ techniques and then go to OBCD.

The simplest direct comparison techniques are the image difference ( $\mathrm{Lu}$ et al., 2005) and image rationing (Howarth, Wickware, 1981). Image regression represents second image as a linear function of first (Lunetta, 1999).

Change vector analysis (CVA) was developed for change detection in multiple image bands (Im and Jensen, 2005; Bayarjargal, 2006). Change vectors are calculated by subtracting pixel vectors of co-registered different-time dates. Principal component analysis (PCA) is applied for change detection in two main ways: applying PCA to images separately and then compare them using differencing or rationing (Richards, 1984) or merging the compared images into one set and then applying the PCA transform (Deng et al, 2008). Tasseled cap transformation (Kauth and Thomas, 1976) produces stable spectral components for long-term studies of forest and vegetation (Rogan et al., 2002; Jin, Sader, 2005). Some other texture-based transforms are developed in (Erener and Düzgün, 2009; Tomowski et al., 2011).

Classification-based change detection contains the postclassification and composite classification. Post-classification comparison presumes that images are first rectified and classified, and then the classified images are compared to measure changes (Im and Jensen, 2005; Bouziani et al., 2010). The supervised (Yuan et al., 2005; Ji et al., 2006; Serpico and Moser, 2006; Castellana et al., 2007; Chatelain et al., 2008; Fernandez-Prieto and Marconcini, 2011) or unsupervised classification (Wiemker, 1997; Melgani and Bazi, 2006; Ghosh et al., 2007; Qi and Rongchun, 2007; Patra et al., 2007; Bovolo et al., 2008; Moser et al., 2011; Subudhi et al., 2014) can be of use. Unfortunately, the errors from classification are propagated into the final change map (Lillesand et al., 2008). In the composite or direct multidate classification (Lunetta, 1999; Lunetta et al., 2006) the rectified multispectral images are stacked together and PCA technique is applied to reduce the number of spectral components.

Machine Learning algorithms are extensively utilized in change detection. Artificial Neural Networks (ANN) are usually trained for generating the complex non-linear regression between input pair of images and output change map (Liu and Lathrop, 2002; Pijanowski et al., 2005). The Support Vector Machine (SVM) approach based on (Vapnik, 2000) considers the finding change and no-change regions as a problem of binary classification in a space of spectral features (Huang et al., 2008; Bovolo et al., 2008). Other machine learning techniques applied for change detection are: decision tree (Im and Jensen, 2005), genetic programming (Makkeasorn et al., 2009), random forest (Smith, 2010), cellular automata (Yang et al., 2008) and deep neural networks (Chu et al., 2016).

Object-based techniques operate with extracted objects. The Direct Object change detection (DOCD) approach is based on the

\footnotetext{
* Corresponding author
} 
comparison of object geometrical properties (Lefebvre et al., 2008; Zhou et al., 2008), spectral information (Miller et al., 2005; Hall and Hay, 2003) or texture features (Lefebvre et al., 2008; Tomowski et al., 2011). In Classified Objects change detection (COCD) approach the extracted objects are compared based on the geometry and class labels (Chant, Kelly, 2009; Jiang and Narayanan, 2003). The framework based on post-classification (Blaschke, 2005) presumes extracting objects and independently classifying them (Im and Jensen, 2005; Hansen and Loveland, 2012). Multitemporal-object change detection presumes that the joint segmentation is performed once for stacked (composite) images (Conchedda et al., 2008; Stow et al., 2008).

In contrary to all these approaches, our technique is based on machine learning and CNN, but it doesn't presume any object classification and performs change detection directly on the image level via GAN.

\section{METHODOLOGY}

In our change detection tasks, we consider image differences that correspond only to the appearance of new or disappearance of existent objects in a scene, rather than differences due to the season specific object changes (see Figure 1), brightness variations and other factors. Such problem of comparing feature domains from different images is solved by using domain adaption and transfer learning approaches, with the best results are delivered by Generative adversarial networks (GAN). As a result, CNN of the same type, namely "pix2pix" (Isola et al., 2017), was selected as a basic CNN model for our change detection method.
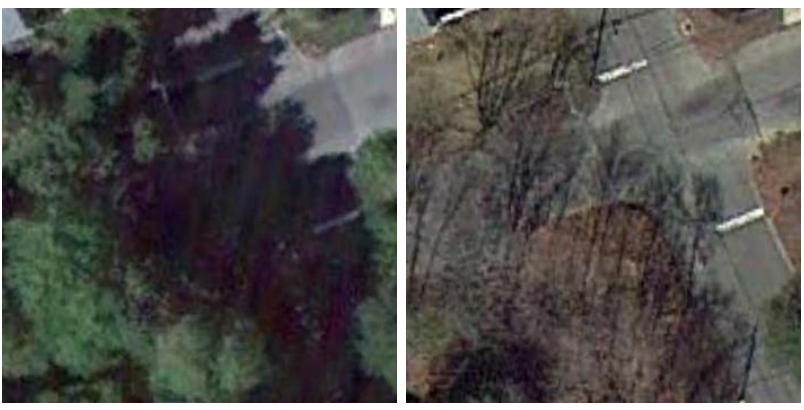

Figure 1. Example of season specific object changes

\subsection{Problem statement}

Conditional GANs learn a mapping from observed image $x$ and random noise vector $z$, to $y$. The main components of a Conditional GAN are two competing neural networks: generator $G$ and discriminator $D$. The generator $G$, on the basis of some space of latent objects features from input data $x$ and a given a priori distribution $p_{z}(z)$, synthesizes output data $y$.

$$
G=G(x, z):\{x, z\} \rightarrow y .
$$

In turn, discriminator $D$ learns to detect "fake" images synthesized by generator $G$ :

$$
D=D(x, y):\{x, y\} \rightarrow[0,1] .
$$

The discriminator maps objects from the data space to $[0,1]$ interval, which is interpreted as the probability that the example was "real".

As a result, $D$ and $G$ play the following two-player minimax game:

$$
G A N=\arg \min _{G} \max _{D} \mathcal{L}_{C G A N}(G, D),
$$

where

$$
\begin{aligned}
& \mathcal{L}_{c G A N}(G, D)=\mathbb{E}_{x, y}[\log D(x, y)]+ \\
& +\mathbb{E}_{x, z}[\log (1-D(x, G(x, z)))],
\end{aligned}
$$

\subsection{Network architectures}

Similarly to pix2pix, our model also contains two main parts: generator and discriminator. A distinctive feature of our implementation is that the generator applies the transformations to a pair of input images simultaneously and extracting features from these images. To do this, the concatenation procedure is applied to the input images of the generator. The generator is based on the "U-Net" network (Ronneberger et al., 2015). It is an encoder-decoder with skip connections between mirrored layers in the encoder and decoder stacks.

The discriminator is based on "PatchGAN" architecture (Isola et al., 2017). In our implementation, the discriminator takes three input images: two images for comparison and one image as a difference map, which can be the output from the generator or ground truth labels. The discriminator learns to distinguish between a difference map synthesized by the generator and ground truth labels. The discriminator structure is quite similar to the encoding part of the generator, but with output as a single value from 0 to 1 . This value evaluates the realistic measure of difference map and the corresponding input images.

To train the discriminator, the generator synthesized the difference map, then the discriminator evaluates whether this difference map is fake or real for two input images. The discriminator parameters are adjusted based on the classification error. The training pipeline of the discriminator is shown on Figure 2.

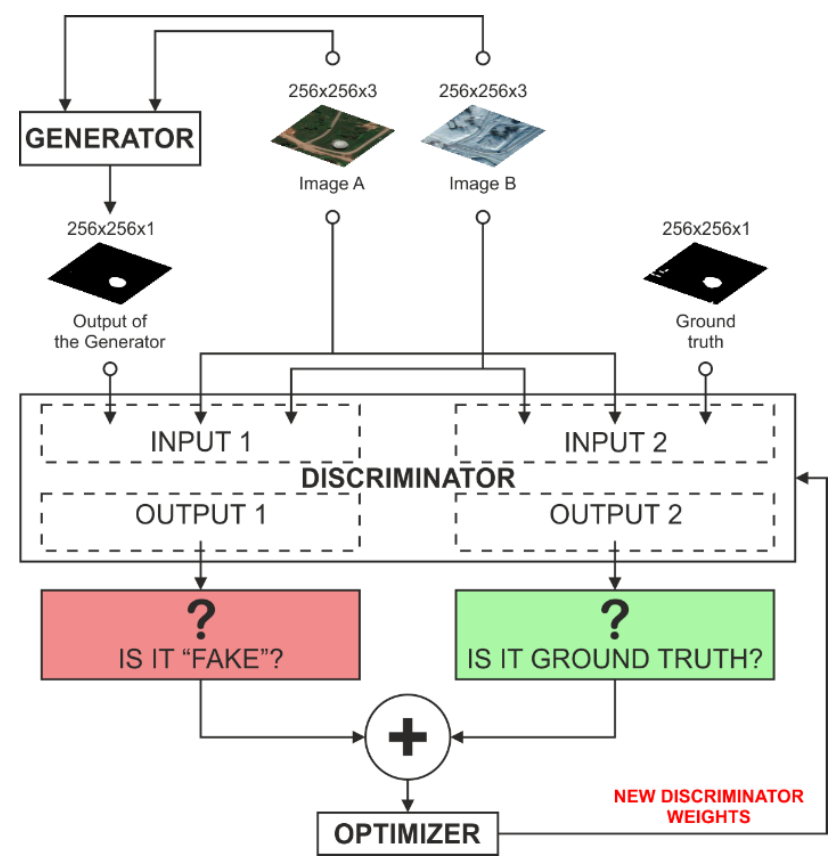

Figure 2. The training pipeline of the discriminator

At the next training step, the generator parameters are updated using classification error using the discriminator output and 
discrepancy between difference map and ground truth labels. The training pipeline of the generator is shown on Figure 3.

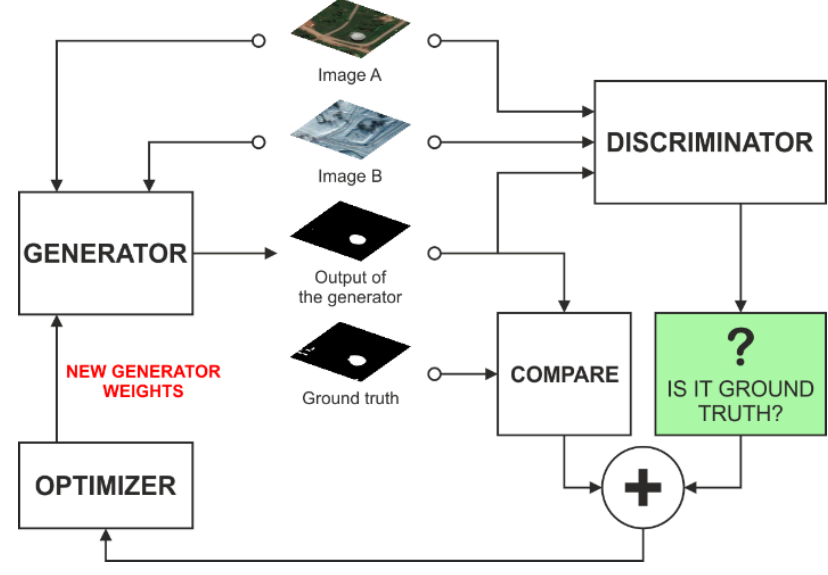

Figure 3. The training pipeline of the generator

In both cases, we use Adam as an optimization algorithm (Kingma et. al., 2015). Our objective is (Isola et al., 2017):

$$
G A N=\arg \min _{G} \max _{D} \mathcal{L}_{C G A N}(G, D)+\lambda \mathcal{L}_{L 1}(G),
$$

where

$$
\mathcal{L}_{L 1}(G)=\mathbb{E}_{x, y, z}\left[\|y-G(x, z)\|_{1}\right]
$$

\section{EXPERIMENTS}

In our study, we adhered to the rule "from simple to complex" in order to better understand how the proposed network stands the challenges. Therefore, we implemented three types of experiments: change detection in synthetic images without objects relative shift, change detection in synthetic images with small relative shift of objects, and change detection in real season-varying remote sensing images.

\subsection{Experiments on the synthetic image dataset without object shifts}

At the first experiment group, we tested performance of our CNN architecture on generated dataset of 12000 triples synthetic images with the dimensions of each image is $256 \times 256$ pixels. The first and second images are an RGB image pair (A and B) with a random homogenous background and random nonintersecting geometric primitives (square, round, rectangle, triangle) of random size and color. The third image is a binary symmetric change detection mask between A and B images. This dataset was split on 8000 training sets and 2000 validation and test sets. An object count limitation is 10 . Some images were smoothed by a Gaussian filter with standard deviation in range $10<\sigma<25$. In addition, some images were noised by additive Gaussian noise with standard deviation in range $10<\sigma<35$. A and B images were smoothed or noised in $20 \%$ of cases of total image count and smoothed at first and then noised in $10 \%$ of cases of total image count.

To evaluate detection results on synthetic images, we used pixel Precision and Recall values, since the difference mask should have a one-to-one correspondence. Precision and recall values during the tests of CNN were 0.95 and 0.96 respectively. Gaussian blur affects the quality of change detection results more than additive Gaussian noise.
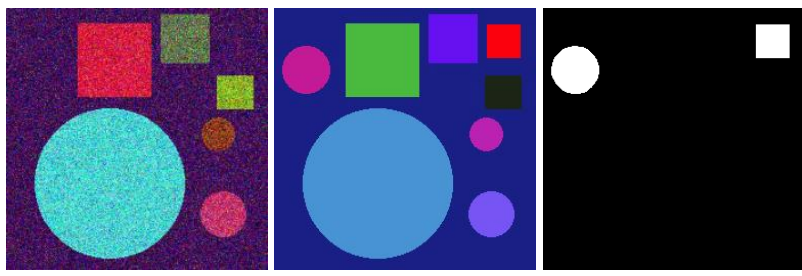

Figure 4. Change detection on synthetic images without objects shifts: left - input image A, middle - input image B, right synthesized difference map

\subsection{Experiments on the synthetic image dataset with object} shifts

Since real images of the earth's surface, obtained at different times by different vision sensors, may have local discrepancies, then in the second type of experiments we tested the proposed network architecture for the case of objects small shifts. Figure 5 shows an example of change detection in case of 5 pixels object shift using the network which was trained on dataset without object shift. To demonstrate that the proposed network architecture can effectively detect changes in case of object shifts, we performed the network fine-tuning. For new training cycle, we created an additional dataset of 12000 triples synthetic images, which contains random shifts of objects represented both on images $\mathrm{A}$ and $\mathrm{B}$. These shifts were in range $[-5,5]$ pixels in horizontal and vertical directions without intersections with the other objects and image boundaries.
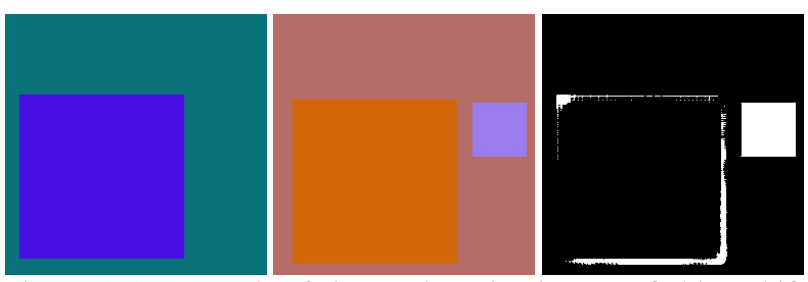

Figure 5. An example of change detection in case of object shift using the network, which was trained on dataset without object shift: left - input image A, middle - input image B, right synthesized difference map.

Precision and recall values during the tests of $\mathrm{CNN}$ were 0.92 and 0.93 respectively. As we noted above, Gaussian blur affects the quality of change detection results more than additive Gaussian noise including case of shifts of geometrical objects. Figure 6 . Shows an example of change detection on synthetic images with small relative shift of objects.
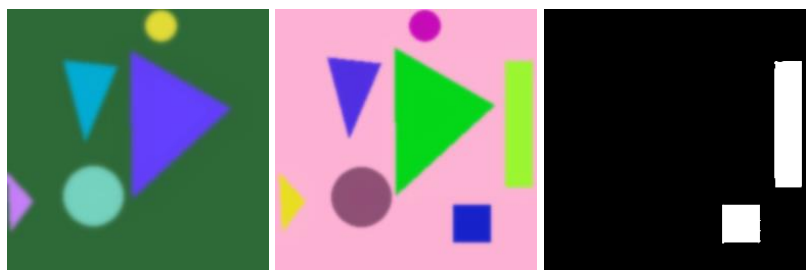

Figure 6. Change detection on synthetic images with small relative shift of objects: left - input image A, middle - input image $\mathrm{B}$, right - synthesized difference map.

\subsection{Experiments on the real image dataset}

At the third type of experiments, the proposed network architecture was evaluated using real images. For dataset generation we used season-varying remote sensing images of the same region, obtained by Google Earth (DigitalGlobe). We obtained 7 pairs of season-varying images with resolution of 
$4725 \times 2700$ pixels for manual ground truth creation and 4 seasonvarying image pairs with minimal changes and resolution of 1900x1000 pixels for adding additional objects manually. Spatial resolution of obtained images was from 3 to $100 \mathrm{~cm} / \mathrm{px}$. That allowed us to take into account objects with different sizes (i.e. from cars to big constructional structures), season changes of natural objects (i.e. from single trees to wide forest areas). Dataset was generated by cropping 256x256 randomly rotated fragments $(0-2 \pi)$ with at least a part of target object. Therefore, object center coordinates were unique and distance between object centers for each axis was 32 pixels. Finally, the dataset contained 16000 image sets with image size 256x256 pixels: 10000 train sets and 3000 test and validation sets.

Due to possible inaccuracy in manual ground truth labeling, we used Intersection over Union (IoU) metrics to assess change detection quality. For IoU calculation, firstly we extract connected regions from ground truth labels and difference map synthesized by the generator. An area is considered to be detected if IoU is greater than some threshold. Then, for the obtained classification values, the average values of Precision and Recall were calculated for the entire test dataset. For IoU thresholds equal to 0.5 , the average Precision and Recall values were 0.26 and 0.32 , respectively. Such low values are associated with poor detection of small sized objects (see Figure 7), to which our network was not originally trained.
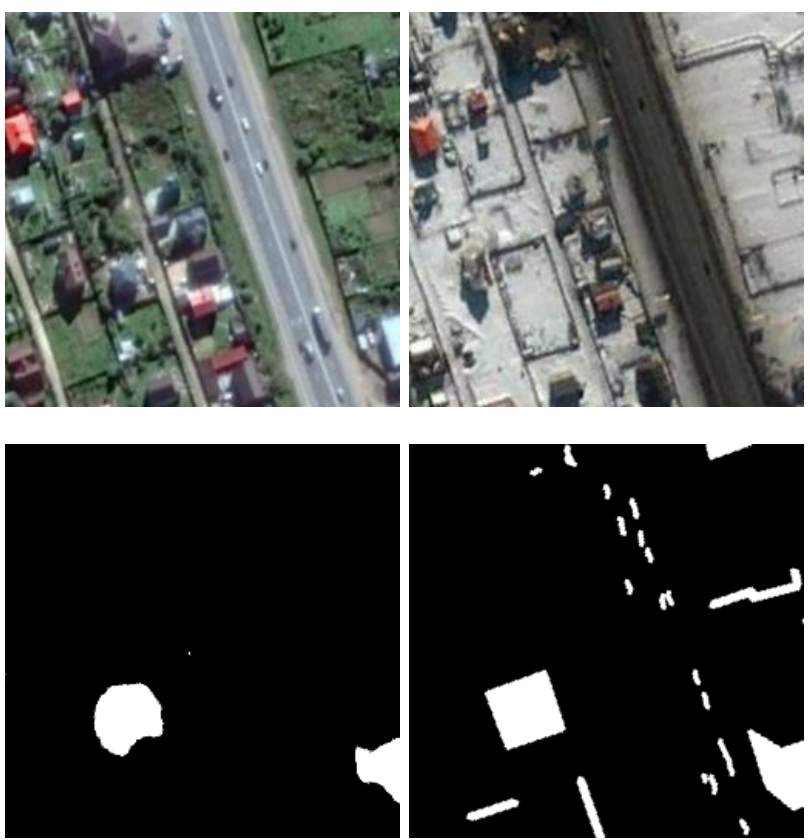

Figure 7. An example of poor detection of small sized objects: top left - input image A, top right - input image B, botton left synthesized difference map, botton right - ground thruth map

Therefore, in the assessment, we did not take into account objects with an area less than 500 pixels. The average Precision and Recall values for this case are shown in Table 1 . The detection of small objects remains the subject of further research. Examples of change detection are shown in Figure 8.

\begin{tabular}{|c|c|c|}
\hline Threshold IoU & Precision & Recall \\
\hline 0.1 & 0.91 & 0.87 \\
0.3 & 0.79 & 0.72 \\
0.5 & 0.72 & 0.65 \\
\hline
\end{tabular}

Table 1. The average Precision and Recall values on the test dataset.
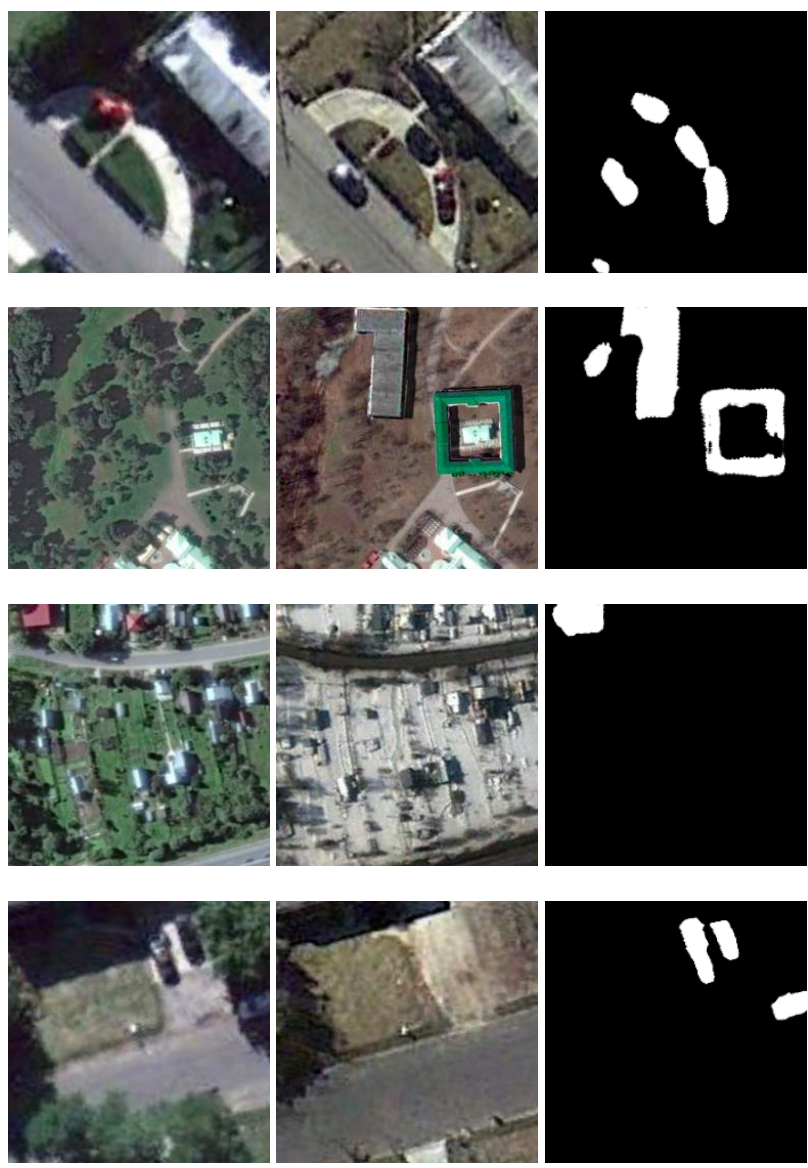

Figure 8. Examples of change detection in remote sensing images: left - input image A, middle - input image B, right synthesized difference map

\section{CONCLUSION}

The paper represents a specially modified Generative adversarial network (GAN) of "pix2pix" architecture for automatic change detection in season-varying remote sensing images. An extensive database of synthetic and real images was created and it will be uploaded for public access. The database contains 12,000 triples of synthetic images without object shift, 12,000 triples of model images with object shift and 16,000 triples of fragments of real remote sensing images. Performed tests have shown that the proposed $\mathrm{CNN}$ is promising and efficient enough in change detection on synthetic and real images.

The created database of synthetic and real images are available for public access at:

https://drive.google.com/file/d/1GX656JqqOyBi_Ef0w65kDGV to-nHrNs9

\section{ACKNOWLEDGEMENTS}

This work was performed with the support of Russian Science Foundation (RNF grant № 16-11-00082).

\section{REFERENCES}

Bayarjargal, Y., Karnieli, A., Bayasgalan, M., Khudulmur, S., Gandush, C., Tucker, C.J., 2006. A comparative study of NOAAAVHRR derived drought indices using change vector analysis. Remote Sensing of Environment, 105, pp. 9-22. 
Benedek, C., Szir'anyi, T., 2009. Change detection in optical aerial images by a multi-layer conditional mixed Markov model. IEEE Trans. Geosci. Remote Sens. 47 (10), 3416-3430.

Blaschke, T., 2005. Towards a framework for change detection based on image objects. Göttinger Geographische Abhandlungen $113,1-9$.

Bouziani, M., Goïta, K., He, D.-C., 2010. Automatic change detection of buildings in urban environment from very high spatial resolution images using existing geodatabase and prior knowledge. ISPRS Journal of Photogrammetry and Remote Sensing, 65, pp. 143-153.

Bovolo, F., Bruzzone, L., Marconcini, M., 2008. A novel approach to unsupervised change detection based on a semisupervised SVM and a similarity measure. IEEE Transactions on Geoscience and Remote Sensing 46, 2070-2082.

Bruzzone, L., Fernandez-Prieto, D., 2002. An adaptive semiparametric and context-based approach to unsupervised change detection in multitemporal remote-sensing images. IEEE Trans. Image Process. 11 (4), 452-466.

Castellana, L., D’Addabbo, A., Pasquariello, G., 2007. A composed supervised/unsupervised approach to improve change detection from remote sensing. Pattern Recogn. Lett. 28 (4), 405413.

Chatelain, F., Tourneret, J.-Y., Inglada, J., March 2008. Change detection in multisensor SAR images using bivariate gamma distributions. IEEE Trans. Image Process. 17 (3), 249-258.

Chant, T.D., Kelly, M., 2009. Individual object change detection for monitoring the impact of a forest pathogen on a hard wood forest. Photogrammetric Engineering \& Remote Sensing, 75, pp. 1005-1013.

Chen, J., Lu, M., Chen, X., Chen, J. Chen, L., 2013. A spectral gradient difference based approach for land cover change detection. ISPRS Journal of Photogrammetry and Remote Sensing, 85, pp. 1-12.

Chu, Y., Cao, G., Hayat, H., 2016. Change Detection of Remote Sensing Image Based on Deep Neural Networks // 2nd International Conference on Artificial Intelligence and Industrial Engineering (AIIE 2016), doi:10.2991/aiie-16.2016.61

Conchedda, G., Durieux, L., Mayaux, P., 2008. An object-based method for mapping and change analysis in mangrove ecosystems. ISPRS Journal of Photogrammetry and Remote Sensing, 63, pp. 578-589.

Deng, J., Wang, K., Deng, Y., Qi, G., 2008. PCA-based land-use change detection and analysis using multitemporal and multisensor satellite data. International Journal of Remote Sensing, 29, pp. 4823-4838

Erener, A., Düzgün, H., 2009. A methodology for land use change detection of high resolution pan images based on texture analysis. Italian Journal of Remote Sensing, 41, pp. 47-59.

Fernandez-Prieto, D., Marconcini, M., Dec 2011. A novel partially supervised approach to targeted change detection. IEEE Trans. Geosci. Remote Sens. 49 (12), 5016-5038.
Ghosh, S., Bruzzone, L., Patra, S., Bovolo, F., Ghosh, A., March 2007. A context-sensitive technique for unsupervised change detection based on Hopfield-type neural networks. IEEE Trans. Geosci. Remote Sens. 45 (3), 778-789.

Hall, O., Hay, G.J., 2003. A multiscale object-specific approach to digital change detection. International Journal of Applied Earth Observation and Geoinformation, 4, pp. 311-327.

Hansen, M.C.; Loveland, T.R., 2012. A review of large area monitoring of land cover change using Landsat data. Remote Sens. Environ. 122, 66-74.

Howarth, P., Wickware, G., 1981. Procedures for change detection using Landsat digital data. International Journal of Remote Sensing, 2, pp. 277-291.

Huang, C., Song, K., Kim, S., Townshend, J.R.G., Davis, P., Masek, J.G., Goward, S.N., 2008. Use of a dark object concept and support vector machines to automate forest cover change analysis. Remote Sensing of Environment, 112, pp. 970-985.

Hussain, M., Chen, D., Cheng, A., Wei, H., Stanley, D., 2013. Change detection from remotely sensed images: From pixelbased to object-based approaches. ISPRS Journal of Photogrammetry and Remote Sensing, 80, pp. 91-106.

Im, J., Jensen, J., 2005. A change detection model based on neighborhood correlation image analysis and decision tree classification. Remote Sensing of Environment, 99, pp. 326-340.

Isola Phillip, Zhu Jun-Yan, Zhou Tinghui, Efros Alexei A., 2017. Image-to-Image Translation with Conditional Adversarial Networks // Computer Vision and Pattern Recognition (CVPR 2017), arXiv:1611.07004.

Ji, W., Ma, J., Twibell, R.W., Underhill, K., 2006. Characterizing urban sprawl using multi-stage remote sensing images and landscape metrics. Computers, Environment and Urban Systems, 30, pp. 861-879.

Jiang, L., Narayanan, R.M., 2003. A shape-based approach to change detection of lakes using time series remote sensing images. IEEE Transactions on Geoscience and Remote Sensing, 41, pp. 2466-2477.

Jin, S., Sader, S., 2005. Comparison of time series tasseled cap wetness and the normalized difference moisture index in detecting forest disturbances. Remote Sensing of Environment, 94, pp. 364-372.

Kauth, R., Thomas, G., 1976. The Tasselled Cap - A Graphic Description of the Spectral-Temporal Development of Agricultural Crops as Seen by LANDSAT, LARS Symposia, 4B, pp.41-51.

Kingma, D. and J. Ba., 2015. Adam: A method for stochastic optimization. // International Conference on Learning Representations (ICLR 2015), arXiv:1412.6980v9.

Lefebvre, A., Corpetti, T., Hubert-Moy, L., 2008. Object-oriented approach and texture analysis for change detection in very high resolution images. In: Geoscience and Remote Sensing Symposium, 2008. IGARSS 2008. IEEE International, pp. $663-$ 666. 
Lillesand, T., Kiefer, R., and J. Chipman, 2008, Remote Sensing and Image Interpretation, 6th ed., New York, NY: John Wiley \& Sons, $756 \mathrm{p}$.

Liu, X., Lathrop, R.G., 2002. Urban change detection based on an artificial neural network. International Journal of Remote Sensing 23, 2513-2518.

Liu, W., Prinet, V., 2006. Probabilistic modeling for structural change inference. In: Asian Conference on Computer Vision. Hyderabad, India, pp. 836-846.

Lu, D., Mauselb. P., Brondízioc, E., Moran, E., 2004. Change detection techniques. Int. J. Remote Sens., 25(12), pp. $2365-$ 2401.

Lu, D., Mausel, P., Batistella, M., Moran, E., 2005. Land-cover binary change detection methods for use in the moist tropical region of the Amazon: a comparative study. International Journal of Remote Sensing 26, 101-114.

Lunetta, R.S., 1999. Applications, project formulation, and analytical approach. In: Lunetta, R.S., Elvidge, C.D. (Eds.), Remote Sensing Change Detection: Environmental Monitoring Methods and Applications. Taylor \& Francis, London, pp. 1-19.

Lunetta, R.S., Knight, J.F., Ediriwickrema, J., Lyon, J.G., Worthy, L.D., 2006. Land-cover change detection using multi-temporal MODIS NDVI data. Remote Sensing of Environment, 105, pp. $142-154$.

Makkeasorn, A., Chang, N.-B., Li, J., 2009. Seasonal change detection of riparian zones with remote sensing images and genetic programming in a semi-arid watershed. Journal of Environmental Management 90, 1069-1080.

Melgani, F., Bazi, Y., Oct 2006. Markovian fusion approach to robust unsupervised change detection in remotely sensed imagery. IEEE Geosci. Remote Sens. Lett. 3 (4), 457-461.

Miller, O., Pikaz, A., Averbuch, A., 2005. Objects based change detection in a pair of gray-level images. Pattern Recognition, 38 , pp. 1976-1992.

Moser, G., Angiati, E., Serpico, S., July 2011. Multiscale unsupervised change detection on optical images by Markov random fields and wavelets. IEEE Geosci. Remote Sens. Lett. 8 (4), 725-729.

Patra, S., Ghosh, S., Ghosh, A., 2007. Unsupervised change detection in remote-sensing images using modified selforganizing feature map neural network. In: International Conference on Computing: Theory and Applications. Washington, DC, USA, pp. 716-720.

Pijanowski, B.C., Pithadia, S., Shellito, B.A., Alexandridis, K., 2005. Calibrating a neural network-based urban change model for two metropolitan areas of the Upper Midwest of the United States. International Journal of Geographical Information Science 19, 197-215.

Qi, Y., Rongchun, Z., 2007. A CMRF-based approach to unsupervised change detection in multitemporal remote-sensing images. In: Int. Conf. on Electronic Measurement and Instruments. Vol. 2. Xi’an, China, pp. 898-904.
Richards, J., 1984. Thematic mapping from multitemporal image data using the principal components transformation. Remote Sensing of Environment, 16, pp. 35-46.

Rogan, J., Franklin, J., Roberts, D., 2002. A comparison of methods for monitoring multitemporal vegetation change using Thematic Mapper imagery. Remote Sensing of Environment. 80, pp. 143-156.

Ronneberger Olaf, Fischer Philipp, Brox Thomas, 2015. U-Net: Convolutional Networks for Biomedical Image Segmentation // Computer Vision and Pattern Recognition (CVPR 2015), arXiv:1505.04597.

Rubis A.Y., Lebedev M.A., Vizilter Y.V., Vygolov O.V., 2016. Morphological image filtering based on guided contrasting. Computer Optics. 40(1):73-79. (In Russ.) DOI:10.18287/24126179-2016-40-1-73-79

Serpico, S., Moser, G., December 2006. Weight parameter optimization by the Ho-Kashyap Algorithm in MRF models for supervised image classification. IEEE Trans. Geosci. Remote Sens. 44 (12), 3695-3705.

Singh, A., 1989. Review article digital change detection techniques using remotely-sensed data. Int. J. Remote Sens., 10(6), pp. 989-1003.

Singh, P., Kato, Z., Zerubia, J., May 2014. A Multilayer Markovian Model for Change Detection in Aerial Image Pairs with Large Time Differences. In: International Conference on Pattern Recognition. Stockholm, Sweden.

Smith, A., 2010. Image segmentation scale parameter optimization and land cover classification using the Random Forest algorithm. Journal of Spatial Science 55, 69-79.

Stow, D., Hamada, Y., Coulter, L., Anguelova, Z., 2008. Monitoring shrubland habitat changes through object-based change identification with airborne multispectral imagery. Remote Sensing of Environment, 112, pp. 1051-1061.

Szir'anyi, T., Shadaydeh, M., Sept 2014. Segmentation of remote sensing images using similarity-measure-based fusion-MRF model. IEEE Geosci. Remote Sens. Lett. 11 (9), 1544-1548.

Subudhi, B. N., Bovolo, F., Ghosh, A., Bruzzone, L., 2014. Spatio-contextual fuzzy clustering with Markov random field model for change detection in remotely sensed images. Optics \& Laser Technology 57, 284 - 292, optical Image Processing.

Tomowski, D., Ehlers, M., Klonus, S., 2011. Colour and Texture Based Change Detection for Urban Disaster Analysis, Urban Remote Sensing Event (JURSE), 2011 Joint, pp. 329-332.

Vapnik, V.N., 2000. The Nature of Statistical Learning Theory, second ed. Springer, New York.

Wiemker, R., 1997. An iterative spectral-spatial bayesian labeling approach for unsupervised robust change detection on remotely sensed multispectral imagery. In: Int. Conf. on Computer Analysis of Images and Patterns. Vol. LNCS 1296. Kiel, Germany, pp. 263-270.

Yang, Q., Li, X., Shi, X., 2008. Cellular automata for simulating land use changes based on support vector machines. Computers \& Geosciences 34, 592-602. 
Yuan, F., Sawaya, K.E., Loeffelholz, B.C., Bauer, M.E., 2005. Land cover classification and change analysis of the Twin Cities (Minnesota) Metropolitan Area by multitemporal Landsat remote sensing. Remote Sensing of Environment, 98, pp. 317-328.

Zhong, P., Wang, R., December 2007. A multiple conditional random fields ensemble model for urban area detection in remote sensing optical images. IEEE Trans. Geosci. Remote Sens. 45 (12), 3978-3988.

Zhou, W., Troy, A., Grove, M., 2008. Object-based land cover classification and change analysis in the baltimore metropolitan area using multitemporal high resolution remote sensing data. Sensors, 8, pp. 1613-1636. 